\title{
Una Propuesta de Gestión de la Incubadora Universitaria de startups en el Perú
}

ArTemio JANQUi GUZMÁN ${ }^{1}$

\begin{abstract}
RESUMEN
Las incubadoras ${ }^{2}$ de empresas en un entorno universitario son ambientes protegidos diseñados para la creación, implementación y desarrollo de startups ${ }^{3}$ de base tecnológica, cuyos resultados de investigación son generados por la comunidad universitaria, propician empleo y crecimiento económico, siendo cada vez más importantes en los países y regiones. El Perú no cuenta con un modelo para una gestión eficaz. Con este estudio se propone un modelo de gestión. El análisis de la literatura y las encuestas al contexto de innovación en el país, identifican que la política de la universidad emprendedora, la dirección con personal de alta calificación, la correcta selección de startups, el adecuado financiamiento y la vinculación universidadempresa-gobierno, son los ejes fundamentales del modelo de gestión de la incubadora universitaria en el Perú, para lograr el éxito de startups.
\end{abstract}

Palabras clave: Incubadora universitaria; startups; universidad emprendedora; ecosistema de innovación; emprendimiento.

A Proposal of the Management of the University InCUbator OF STARTUPS IN Peru

\section{ABSTRACT}

Business incubators in a university area are protected environments designed for the creation, implementation and startups develop of technology base, whose research results are generated for the university community, promote employment and economic increase, being every time more important in the countries and regions. Peru doesn't have a model for an effective management. With this study proposes a management model. The analysis of the literature and the surveys on the context of innovation in the country, identify that the policy of entrepreneurial university, the management with highly qualified staff, the correct selection of startups, the adequate financing and the university-businessgovernment link, are the fundamental axes of the management model of the university incubator in Peru, for achieve the startups success.

Keywords: University incubator; startups; entrepreneurial university; innovation ecosystem; entrepreneurship.

\section{INTRODUCCIÓN}

Las incubadoras de empresas son alternativas ideadas para crear un ambiente de protección a la creación e implementación de nuevas empresas. La incubadora de empresas universitarias (UBI), es la organización diseñada para acelerar el desarrollo económico nacional, que ayuda a las startups, en particular a las NTBFs (nuevas empresas de base tecnológica), durante su fase de crecimiento y desarrollo (Studdard, 2006); para Chindaprasert et al. (2006), es un mecanismo que desarrolla la universidad para obtener los resultados de la investigación, incluyendo las invenciones e innovaciones para la comercialización con éxito a través de la creación de nuevas empresas; son instrumentos que promueven la creación de startups de base tecnológica quienes surgen de los resultados de investigación generados por la comunidad científica (Wonglimpiyarat, 2016). Las startups se convierten cada vez más en una fuente importante de creación de empleos, en particular en los períodos de desaceleración económica y que dan lugar a una amplia gama de iniciativas de política pública; la puesta en marcha de programas de emprendimiento, implementados sobre todo en instituciones de educación superior, apoyan el desarrollo de incubadoras universitarias para: la eliminación o disminución de obstáculos regulatorios en la creación de empresas, la mejora de los regímenes de competencia con el fin de facilitar la entrada de nuevas empresas, las reformas de los mercados de capital para simplificar el acceso de nuevas empresas al financiamiento, así como la apertura de nuevas líneas de crédito, el otorgamiento de préstamos subsidiados o la aplicación de esquemas de garantías para nuevas empresas ofrecidas por los bancos de desarrollo (OECD, 2010a). En la actualidad el desarrollo de startups basadas en la innovación tecnológica se ha convertido cada vez importante en las economías competitivas, como participe en el desarrollo social y económico de los países, las incubadoras dirigen diversas industrias como la biotecnología, la energía limpia, tecnologías

1 Maestro en Gerencia de Proyectos de Ingeniería, Profesor del Departamento Académico de Ingeniería Eléctrica, Universidad Nacional de San Antonio Abad del Cusco. Perú. ORCID: https://orcid.org/0000-0002-9191-8364

E-mail: artemio.janqui@unsaac.edu.pe

2 Las incubadoras son herramientas para impulsar el nacimiento de empresas, pues ofrecen la asistencia necesaria para crecer. Existen dos modelos de incubación, uno enfocado a desarrollar un producto para una gran empresa y otro dirigido a lanzar un producto directamente al mercado.

3 Una Startup es un término utilizado para definir a aquellas empresas que se encuentran en edad temprana o de nueva creación y presentan grandes posibilidades de crecimiento. 
de cerámica, Internet, software y telecomunicaciones, alta tecnología y las artes: el concepto es que las empresas tengan acceso a los clientes, a los recursos externos, al cocimiento, y la legitimidad, cumpliendo con la calidad, la innovación, la I+D (Bruneel, 2012).

En las incubadoras universitarias, hace falta comprender la función de relaciones y ventajas mutuas derivadas de vincular el talento, la tecnología, el capital y el know-how para aprovechar el talento empresarial, acelerar el desarrollo de nuevas empresas y la comercialización de la tecnología (Grimaldi y Alessandro, 2005); tanto en los países desarrollados y en desarrollo, existen barreras para la creación y expansión de las startups, debido principalmente a los altos riesgos e incertidumbre en sus operaciones (OECD, 2013) y como tal, hay necesidad de identificar y conocer cuáles son los factores para que las startups logren el éxito y la supervivencia en las diferentes etapas de su desarrollo. En ese contexto se han desarrollado diversos trabajos de procedimientos y gestión en las incubadoras, donde se identifican las dimensiones y factores que influyen la gestión de las incubadores de empresas; entre ellos Matt y Feng (2010), para quienes la gestión de la incubadora se sustenta en la relación de los objetivos y resultados. Para Somsuk y Laosirihongthong (2014), la gestión de las incubadoras debe ser estratégica basado en el manejo eficiente de cuatro dimensiones: recursos humanos, recursos tecnológicos, recursos financieros, recursos de organización.

De la búsqueda de la literatura, solo se ha encontrado en el trabajo de Bergek y Norrman (2008), quienes desarrollan el modelo de gestión con enfoque centrado en los resultados y en la incertidumbre, muestra correlación directa entre las fases del proceso y el rendimiento de la incubadora; proponen tres componentes: la selección de startups, como un factor de éxito; el apoyo a las startups, asociado con las diversas actividades intangibles; la mediación, referido a la forma en que la incubadora relaciona a las startups incubados entre sí y con el mundo exterior, en busca y logro de todo tipo de recursos. Además, en todas las regiones, es probable que haya diferencias en los modelos de incubación universitaria y en el rendimiento, este enfoque contrasta con un mejor espíritu de práctica universal aplicable en todas las regiones que sugiere la necesidad de identificar y aprovechar las influencias regionales únicas e idiosincrásicas en los modelos de incubación universitaria (Daskalopoulou et al., 2010). Por consiguiente, no se ha encontrado un estudio que propone la gestión eficaz de la incubadora universitaria de startups de base tecnológica, que englobe la configuración y el proceso de incubación, y tampoco explican su relación con el éxito de las startups en las diferentes etapas de su desarrollo, sobre todo en países emergentes y en desarrollo.

En ese sentido, el presente trabajo tiene como objetivo llenar este vacío. A partir del estudio de la literatura y la comparación conceptual de principales modelos de gestión de incubadoras, se desarrolla y considera en el constructo del marco conceptual de gestión eficaz el emprendimiento universitario, las políticas del entorno, conocimientos sobre equipos y estructuras dinámicas, manejo de recursos con valor, para identificar los factores predominantes que influyen el éxito de la incubación de startups de base tecnológica, y realizado un diagnóstico del ecosistema de incubación de startups en el Perú, se propone como un acercamiento preliminar sujeto a futuras investigaciones de un modelo de gestión eficaz de la incubadora universitaria de startups en el Perú. Utilizando la herramienta scamper que permite la adaptación, se identifica los factores de éxito: una política de universidad emprendedora, equipo directivo con personal de alta calificación, correcta selección de startups, mecanismos adecuados de financiamiento y la vinculación universidad-empresa-gobierno, como ejes para una gestión eficaz.

El trabajo se organiza en seis secciones. la segunda sección fundamenta la base teórica a partir del estado del arte, la tercera explica la metodología del estudio y los hallazgos, la cuarta los resultados, la quinta propone el modelo de gestión de la incubadora universitaria de startups en el Perú, y finalmente en la sexta, presentar la discusión, las implicancias, limitaciones y conclusión del estudio.

\section{REVISIÓN DE LA LITERATURA}

La incubadora de empresas es un mecanismo que ofrece un enfoque estratégico de valor añadido, alberga startups para ayudarles a desarrollarse, se comporta como una herramienta catalizadora para el desarrollo económico, proporciona una amplia gama de recursos y servicios y prácticas de gestión; la incubadora de empresas universitarias (UBI) es un mecanismo que desarrolla la universidad para obtener los resultados de la investigación, incluyendo la creación de nuevas empresas.

En las incubadoras de empresa se practican modelos de gestión con diferentes estructuras y procedimientos. Grimaldi y Alessandro (2005), proponen dos modelos de gestión, sus características de ser públicos o privados, con estrategia en especialización de 
servicios satisfactorios para las empresas. Bergek y Norrman (2008), desarrollan un modelo basado en el proceso de evaluar la gestión mediante el rendimiento relacionando los objetivos y los resultados. Para Matt y Feng (2010), la gestión de la incubadora universitaria es para crear startups exitosos, basados en la innovación de los recursos tangibles e intangibles. Somsuk y Laosirihongthong (2014), proponen un modelo de gestión estratégica para el éxito de la incubadoras de empresas universitarias, este modelo tiene como base el manejo eficiente de los recursos sustentado en la teoría de los recursos RBV y su aplicación, que asegura la supervivencia, el crecimiento y la ventaja competitiva; además explican cómo los recursos y capacidades de la incubadora universitaria permiten a las startups, a la universidad patrocinadora, a una comunidad o incluso a la incubadora en sí, ganar ventaja competitiva y un alto rendimiento. Rubin et al. (2015), analizan incubadoras de empresas en los contextos de Israel y Australia, países que conforman la OCDE; proponen para estos casos, un modelo de gestión basado en la interrelación de las empresas incubadas; consideran a la incubadora como una organización y a las empresas incubadas como unidades de negocio, y para mejorar el rendimiento, distinguen tres tipos de interrelaciones y los denominan: portador de conocimiento tecnológico; portador de conocimiento del mercado; y portador de recursos financieros. McAdam et al. (2016), consideran que en el modelo de incubación de la triple hélice (academia, industria y los actores gubernamentales regionales) debe incluirse a los usuarios como una cuarta hélice, porque son vistos como un grupo de la sociedad que tienen intereses específicos, y el nivel de su compromiso puede variar de acuerdo con el tipo de universidad y región. demuestran que los modelos de incubación dentro de una región están influenciados: 1) por el entorno macro, que es el contexto regional que dicta la prioridad de relevancia de los interesados y los recursos disponibles para la incubación; 2) el entorno meso, la universidad donde los factores de su organización determinan su tipo (es decir, la cultura, la misión, estrategia, recursos, habilidades, conocimientos), tendrán un impacto en el modelo de incubación adoptada; 3) el entorno micro, la incubadora, que representa los procesos de incubación reales que son el resultado de la colaboración e interacción de los interesados. Pauwels et al. (2016), proponen una estructura del modelo de gestión del acelerador, que contiene cinco parámetros o factores clave: el Paquete de programas, comprende los servicios que presta el acelerador a sus incubados; el Enfoque estratégico, se refiere a las decisiones estratégicas del acelerador; el Proceso de selección, referido a un riguroso proceso de selección de múltiples etapas en un período de tiempo; la Estructura de financiación, referido a los programas reciben gran parte de su capital; y las Relaciones con exalumnos, se refiere a mantener relaciones estrechas y activas con las empresas que se gradúan e sus programas. Baraldi y Ingemansson (2016), proponen un modelo en base a seis "conductores estratégicos". Correia da Silva et al. (2016), sostienen que la gestión se basa en el concepto estratégico, además la incubadora de empresas tiene diferentes percepciones que afecta la "asertividad". (Wonglimpiyarat, 2016) propone un modelo de gestión de las incubadoras de empresas universitarias, para la comercialización de tecnología, basado en : una fuerte interacción entre la academia, la industria y el gobierno, quienes apoyan con estímulos (becas, subvenciones, incentivos fiscales, créditos fiscales por actividades de innovación, etc.), incentivos económicos y fiscales (para mejorar la explotación y promover la comercialización del IP, la deducción fiscal del $200 \%$ de los gastos en I + D); propiciar una estrecha coordinación en la comercialización y la transferencia de tecnología universitaria, en colaboración con las agencias gubernamentales y los parques de ciencias tecnologías/parques de innovación; actuar como intermediarios para asesorar y ayudar a las startups al acceso a fuentes alternativas de financiación.

Respecto a dimensiones. Somsuk y Laosirihongthong (2014), proponen un modelo de gestión basado en los criterios de su rendimiento y logran clasificar en cuatro las dimensiones de recursos: 1) los recursos humanos, se refiere a los atributos del talento y habilidades; 2) los recursos tecnológicos, se refieren a los productos, capacidades y tecnologías altamente especializadas; 3) los recursos financieros, se refieren a todo el apoyo financiero y en especie que las empresas pueden utilizar; 4) los recursos de organización, se refieren a las capacidades asociadas a la planificación, control, coordinación, sistemas, rutinas y relaciones incrustadas en la empresa. Baraldi y Ingemansson (2016), consideran una integración más amplia con los actores específicos con los que las incubadoras interactúan e identifican siete componentes claves:1) Lugar, comprende la ubicación física de la incubadora;2) Tiempo, que la incubadora contribuye con el proceso empresarial a sus incubados;3) Fuentes, se refiere a las ideas estrictamente científicas procedentes de una universidad;4) Recursos, proporción de recursos tangibles e intangibles a los inquilinos;5) Control/gobernanza, es un control directo sobre sus empresas incubadas a través de su función de supervisión;6) Actividades/servicios, son los servicios prestados como las actividades de la selección, el 
apoyo empresarial, acceso a la financiación y la mediación;7) Resultados se refiere a los valores creados internamente por la incubadora.

En cuanto a factores. Grimaldi y Alessandro (2005), definen factores: misión/estrategia institucional, sector industrial, ubicación, mercado, origen de las ideas, fase de intervención, período de incubación, fuentes de ingresos, servicios ofrecidos, equipo de gestión. En la Tabla 1, se muestra el resumen de diferentes estudios sobre modelos, agrupados por el sustento en: procedimientos de gestión, dimensiones y factores que influyen la gestión de la incubadora.

\section{METODOLOGÍA}

Desde el punto de vista metodológico, se trata de una investigación cualitativa y cuantitativa llevada a efecto entres partes, descrita y esquematizada cada uno de sus acápites en la siguiente figura 1.
Las organizaciones involucradas en la obtención, organización, análisis e interpretación de datos de la investigación, se muestran en las Tablas 2 y 3 , son activos participantes del contexto de incubación en el Perú, se aplicaron dos tipos de encuestas. El primero a los responsables de las incubadoras de empresas, según la base de datos del programa piloto de formación de gerentes de incubadoras de empresas del año 2016, a cargo de PRODUCE, institución adjunta al Ministerio de la Producción.

El segundo a los responsables de los startups, véase la Tabla 3, según la base de datos del año 2016 del programa STARTPERU, institución adjunta al Ministerio de la Producción.

En ambos casos, mediante la herramienta "Google Forms", los correos electrónicos de los encuestados, el seguimiento mediante visitas directas y llamadas telefónicas en dos etapas: la primera encuesta de

Tabla 1. Modelos de gestión de las Incubadoras de Empresas

\begin{tabular}{|c|c|}
\hline \multicolumn{2}{|c|}{ Procedimientos y gestión de las incubadoras de empresas } \\
\hline \multicolumn{2}{|c|}{ Modelos de gestión con diferentes estructuras y procedimientos } \\
\hline Grimaldi y Alessandro (2005) & $\begin{array}{l}\text { Proponen dos modelos de gestión, sus características de ser públicos o privados, con estrate- } \\
\text { gia en especialización de servicios satisfactorios para las empresas }\end{array}$ \\
\hline Bergek y Norrman (2008) & $\begin{array}{l}\text { Desarrollan un modelo basado en el proceso de evaluar la gestión mediante el rendimiento } \\
\text { relacionando los objetivos y los resultados }\end{array}$ \\
\hline Matt y Feng (2010) & $\begin{array}{l}\text { La gestión de la incubadora universitaria es para crear startups exitosos, basados en la innova- } \\
\text { ción de los recursos tangibles e intangibles. }\end{array}$ \\
\hline Somsuk y Laosirihongthong (2014) & Proponen un modelo de gestión estratégica basado en el manejo eficiente de los recursos \\
\hline \multicolumn{2}{|c|}{ Modelos de gestión para cada tipo de incubadora mediante los recursos humanos específicos } \\
\hline Rubin et al. (2015) & Plantean un modelo de gestión basado en la interrelación de las empresas incubadas \\
\hline McAdam et al. (2016) & Consideran el modelo de incubación de la triple hélice \\
\hline Pauwels et al. (2016) & Proponen el modelo de gestión del acelerador con cinco parámetros \\
\hline Baraldi y Ingemansson (2016) & Proponen un modelo en base a seis "conductores estratégicos" \\
\hline Correia da Silva et al. (2016) & $\begin{array}{l}\text { Sostienen que la gestión se basa en el concepto estratégico, además la incubadora de empre- } \\
\text { sas tiene diferentes percepciones que afecta la "asertividad". }\end{array}$ \\
\hline \multicolumn{2}{|c|}{ Dimensiones y factores que influyen la gestión de las incubadoras de empresas } \\
\hline \multicolumn{2}{|l|}{ Dimensiones } \\
\hline Somsuk y Laosirihongthong (2014) & $\begin{array}{l}\text { Clasifican cuatro dimensiones principales: recursos humanos, recursos tecnológicos, recursos } \\
\text { financieros, recursos de organización. }\end{array}$ \\
\hline Baraldi y Ingemansson (2016) & $\begin{array}{l}\text { Identifican siete componentes claves: lugar, tiempo, fuentes, recursos, control/gobernanza, } \\
\text { actividades/servicios, resultados. }\end{array}$ \\
\hline \multicolumn{2}{|l|}{ Factores } \\
\hline Grimaldi y Alessandro (2005) & $\begin{array}{l}\text { Definen factores: misión/estrategia institucional, sector industrial, ubicación, mercado, origen de } \\
\text { las ideas, fase de intervención, período de incubación, fuentes de ingresos, servicios ofrecidos, } \\
\text { equipo de gestión. }\end{array}$ \\
\hline \multicolumn{2}{|c|}{$\begin{array}{l}\text { En resumen, el estudio y análisis de la literatura permite identificar cinco dimensiones: Organización, factor selección de los startups; } \\
\text { Tecnología, factor idea innovadora del producto; Financiamiento, soporte consultoría; Recursos Humanos, factor gerentes talentosos con } \\
\text { experiencia; Comercialización, factor vinculación y colaboración con instituciones que relacionan la universidad-empresa-gobierno. }\end{array}$} \\
\hline
\end{tabular}

Fuente: Elaboración propia 


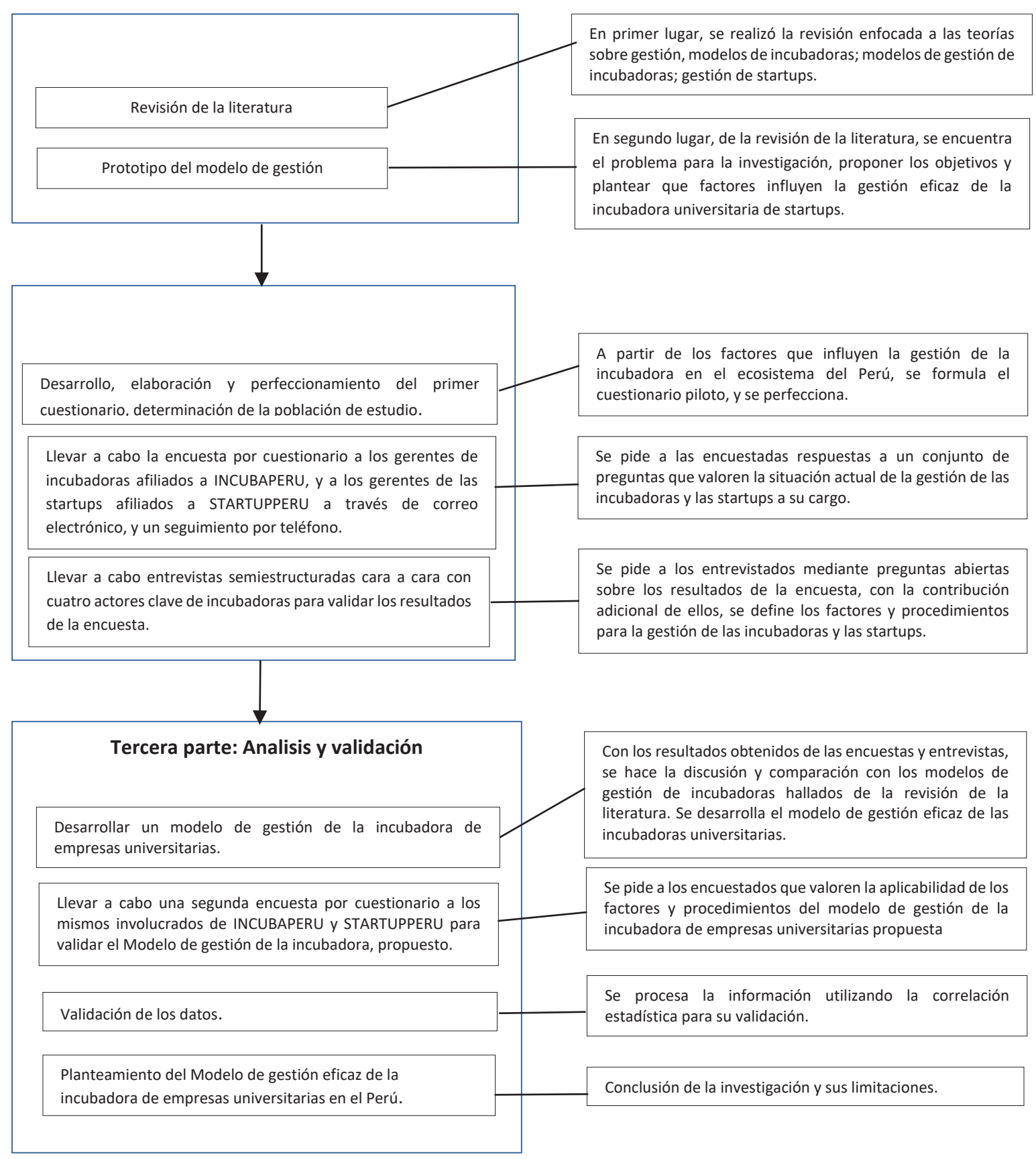

Figura 1. Esquema que grafica la metodología de la investigación.

Fuente: Elaboración propia.

enero - abril de 2017 y la segunda encuesta de enero - febrero de 2019; las informaciones obtenidas se procesaron mediante las herramientas del Excel y el SPSS statistics22. La información obtenida, permite plantear la siguiente proposición:

Proposición: Para que la gestión de la incubadora universitaria de startups sea eficaz y logre el éxito, la incubadora debe desarrollar y practicar políticas de sinergia universitaria; una dirección con talento y emprendedor en conjunto; una selección óptima de sus inquilinos; un mecanismo viable de financiamiento; y políticas viables para la comercialización de la innovación tecnológica universitaria.

A base de preguntas cerradas en una escala de Likert de 1 a 4, los expertos encuestados evaluaron el "Modelo de gestión de la incubadora universitaria de startups en el Perú" de 5 variables, asimismo 
Tabla 2. Incubadoras de empresas consideradas en la investigación

\begin{tabular}{|c|c|c|c|c|}
\hline id & Nombre de la Incubadora & Institución a la que pertenece & Fecha de constitución & Fecha encuesta \\
\hline 1 & BIOINCUBA ** & Universidad Particular Cayetano Heredia & $10 / 02 / 2008$ & $\begin{array}{ll}1^{\circ}: & 16 / 02 / 2017 \\
2^{\circ}: & 27 / 02 / 2019\end{array}$ \\
\hline 2 & KAMAN ** & Universidad Católica San Pablo & $1 / 01 / 2015$ & $\begin{array}{ll}1^{\circ}: & 20 / 02 / 2017 \\
2^{\circ}: & 5 / 02 / 2019\end{array}$ \\
\hline 3 & $\begin{array}{l}\text { Centro De Innovación Y Desarrollo } \\
\text { Emprendedor } \\
\text { CIDE PUCP }{ }^{* *}\end{array}$ & $\begin{array}{l}\text { Pontificia Universidad Católica del Perú } \\
\text { PUCP }\end{array}$ & $1 / 06 / 1995$ & $\begin{array}{ll}1^{\circ}: & 27 / 02 / 2017 \\
2^{\circ}: & 18 / 02 / 2019\end{array}$ \\
\hline 4 & WAYRA PERÚ * & Telefónica Open Future & $21 / 10 / 2011$ & $1^{\circ}: \quad 28 / 02 / 2017$ \\
\hline 5 & $\begin{array}{l}\text { Centro de Innovación y Emprendi- } \\
\text { miento de la Universidad Nacional de } \\
\text { Trujillo - INCUBA UNT }\end{array}$ & Universidad Nacional de Trujillo & $1 / 05 / 2016$ & $\begin{array}{ll}1^{\circ}: & 28 / 03 / 2017 \\
2^{\circ}: & 31 / 01 / 2019\end{array}$ \\
\hline 6 & $\begin{array}{l}1551 \text { UNMSM Incubadora de Empre- } \\
\text { sas Innovadoras ** }\end{array}$ & $\begin{array}{l}\text { Universidad Nacional Mayor de San } \\
\text { Marcos }\end{array}$ & $15 / 09 / 2015$ & $\begin{array}{ll}1^{\circ}: & 28 / 03 / 2017 \\
2^{\circ}: & 20 / 02 / 2019\end{array}$ \\
\hline 7 & INCUBAGRARIA ** & Universidad Nacional Agraria la Molina & $1 / 01 / 2016$ & $\begin{array}{ll}1^{\circ}: & 29 / 03 / 2017 \\
2^{\circ}: & 1 / 02 / 2019\end{array}$ \\
\hline 8 & $\begin{array}{l}\text { Incubadora de Negocios de Base } \\
\text { Tecnológica, } \\
\text { STARTUP UNI ** }\end{array}$ & Universidad Nacional de Ingeniería & $6 / 06 / 2016$ & $\begin{array}{ll}1^{\circ}: & 7 / 04 / 2017 \\
2^{\circ}: & 8 / 02 / 2019\end{array}$ \\
\hline 9 & INCUBACIÓN UP ** & Universidad del Pacífico & $1 / 01 / 2015$ & $\begin{array}{ll}1^{\circ}: & 9 / 04 / 2017 \\
2^{\circ}: & 4 / 02 / 2019 \\
\end{array}$ \\
\hline 10 & USIL VENTURES * & Universidad San Ignacio de Loyola & $1 / 02 / 2016$ & $1^{\circ}: \quad 26 / 04 / 2017$ \\
\hline 11 & ANDES ACCELERATOR ** & Universidad San Martín de Porres & $12 / 02 / 2016$ & $\begin{array}{ll}1^{\circ}: & 26 / 04 / 2017 \\
2^{\circ}: & 18 / 02 / 2019\end{array}$ \\
\hline
\end{tabular}

Fuente: Elaboración propia ("participa en primera encuesta, ${ }^{* *}$ participa en las dos encuestas)

Tabla 3. Startups considerados en la investigación

\begin{tabular}{|l|l|l|l|l|l|}
\hline Id & \multicolumn{1}{|c|}{ Nombre de la Startups } & $\begin{array}{c}\text { Incubadora a la } \\
\text { que pertenece }\end{array}$ & \multicolumn{1}{|c|}{$\begin{array}{c}\text { Fecha de } \\
\text { Constitución }\end{array}$} & $\begin{array}{l}\text { Fecha de } \\
\text { Afiliación }\end{array}$ & \multicolumn{1}{|c|}{$\begin{array}{c}\text { Fecha de } \\
\text { encuesta }\end{array}$} \\
\hline 1 & RIQRA & CIDE-PUCP & $1 / 12 / 2015$ & $15 / 1 / 2016$ & $1^{\circ}: 23 / 01 / 2017$ \\
\hline 2 & INNGRESA & CIDE-PUCP & $1 / 7 / 2015$ & $1 / 6 / 2016$ & $1^{\circ}: 23 / 01 / 2017$ \\
\hline 3 & STRATEGIO & CIDE PUCP & $11 / 11 / 2013$ & $12 / 5 / 2015$ & $1^{\circ}: 23 / 01 / 2017$ \\
\hline 4 & AUTODIAGNOSTICO & UTEC Ventures & $11 / 6 / 2014$ & $1 / 12 / 2015$ & $1^{\circ}: 23 / 01 / 2017$ \\
\hline 5 & ROCKO TOOLS LAB & UTEC Ventures & $15 / 1 / 2014$ & $14 / 1 / 2015$ & $1^{\circ}: 23 / 01 / 2017$ \\
\hline 6 & NIXDEN & UTEC Ventures & $26 / 9 / 2015$ & $25 / 5 / 2017$ & $1^{\circ}: 23 / 01 / 2017$ \\
\hline 7 & BIONDI ** & BIOINCUBA & $1 / 1 / 2015$ & $1 / 1 / 2015$ & $\begin{array}{l}1^{\circ}: 24 / 01 / 2017 \\
2^{\circ}: 23 / 01 / 2019\end{array}$ \\
\hline 8 & FURNITURE Carpintería Digital & 1551 UNMSM & $5 / 12 / 2014$ & $23 / 4 / 2015$ & $1^{\circ}: 24 / 01 / 2017$ \\
\hline 9 & TULLPI & UTEC Ventures & $27 / 12 / 2014$ & $15 / 1 / 2015$ & $1^{\circ}: 24 / 01 / 2017$ \\
\hline 10 & MAQUINA DE CORTE CON TECNOLOGIA -CNC & BIOINCUBA & $4 / 1 / 2016$ & $11 / 1 / 2016$ & $1^{\circ}: 27 / 01 / 2017$ \\
\hline 11 & WIWA & UTEC Ventures & $1 / 6 / 2016$ & $27 / 7 / 2016$ & $1^{\circ}: 9 / 02 / 2017$ \\
\hline 12 & HAVENTURAS & INCUBA-UNT & $7 / 9 / 2016$ & $21 / 1 / 2017$ & $1^{\circ}: 11 / 04 / 2017$ \\
\hline 13 & MI TIENDA & No indican & No indican & No indican & $2^{\circ}: 23 / 01 / 2019$ \\
\hline 14 & VIDA SOFFWARE & No indican & No indican & No indican & $2^{\circ}: 20 / 02 / 2019$ \\
\hline 15 & INNGRESA S.A.C. & No indican & No indican & No indican & $2^{\circ}: 20 / 02 / 2019$ \\
\hline 16 & UDOCZ & No indican & No indican & No indican & $2^{\circ}: 20 / 02 / 2019$ \\
\hline 17 & NOOVA7 & No indican & No indican & No indican & $2^{\circ}: 20 / 02 / 2019$ \\
\hline 18 & CHASKI & No indican & No indican & No indican & $2^{\circ}: 20 / 02 / 2019$ \\
\hline 19 & CIVILSIG & No indican & No indican & No indican & $2^{\circ}: 23 / 02 / 2019$ \\
\hline 20 & BIOAQUA NUTRITION & No indican & No indican & No indican & $2^{\circ}: 24 / 02 / 2019$ \\
\hline
\end{tabular}

Fuente: Elaboración propia (** participa en las dos encuestas) 
validaron estadísticamente la proposición mediante el modelo de regresión y correlación de Pearson mostrado en la Tabla 4.

La correlación de Pearson de Rp $=0.817$, y un Valor $p=0.002<0.05$ indica la aceptación de la proposición, que una gestión eficaz de la incubadora universitaria de startups logra el éxito, si la incubadora desarrolla y practica políticas de sinergia universitaria; una dirección con talento y emprendedor en conjunto; una correcta selección de sus inquilinos; un mecanismo viable de financiamiento; y políticas viables para la comercialización de la innovación tecnológica universitaria.

\section{RESULTADOS}

\section{Factores que influyen el modelo de gestión de la incubadora de empresas}

Se infiere que una política de universidad emprendedora basada en la fuerte vinculación interna influye positivamente en la creación e incubación exitosa de las startups. Diferentes autores a quienes se considera expertos en el tema, en sus trabajos de investigación encuentran cuales son los factores influyentes para la gestión, y las conclusiones a las que han llegado, tienen validez empírica, entre ellos:

Respecto a las políticas universitarias, Kalar y Antoncic (2015), Wonglimpiyarat (2016), Stal et al. (2016), Good et al. (2019), identifican los factores que influyen la creación de un ambiente emprendedor estos son: emprendimiento universitario, componentes estratégicos a desarrollar, y actividades que estimulan a los estudiantes al emprendimiento.

Se encontró evidencias porque Grimaldi y Alessandro (2005), Bergek y Norrman (2008), Soltanifar y Keramati (2012), Somsuk y Laosirihongthong (2014), Yong et al. (2018), sostienen que las organizaciones del siglo XXI dependen en gran medida de los equipos y estructuras de trabajo, de un entorno dinámico e hipercompetitivo para la gestión eficiente, un factor crítico es el equipo de gestión, identifican el perfil del director y los conformantes del equipo, sus competencias, y factores e indicadores de la gestión del equipo.

Del estudio de Bergek y Norrman (2008), Mat y Feng (2010), Bruneel et al. (2012), Somsuk y Laosirihongthong (2014), Pauwels et al. (2016), Stal et al. (2016), Mrkajic (2017), se encuentra evidencia, que un factor crítico es la selección de los inquilinos y su procedimiento, porque la selección permite a las incubadoras entregar eficazmente recursos con valor a las empresas.

En los trabajos de Matt y Feng (2010), Somsuk y Laosirihongthong (2014), Rubin et al. (2015), Wonglimpiyarat (2016), Arena et al. (2018), se encuentra que el financiamiento de la investigación y desarrollo, la innovación y el emprendimiento está relacionando estrechamente con las políticas y objetivos de la gestión. Ankrah y AL-Tabbaa (2015), Rajalo y Vadi (2017), Liu y Huang (2018), encuentran que la universidad, la empresa y el gobierno, tienen como objetivo, promover el crecimiento económico sostenible, la comercialización de la innovación tecnológica universitaria y la competitividad de los países.

Por consiguiente, se infiere que una política de universidad emprendedora basada en la fuerte vinculación interna influye positivamente en la creación e incubación exitosa de las startups; el equipo de personal con talento y experiencia influye positivamente al éxito en conjunto de la incubadora y las

Tabla 4. Determinación de Correlaciones mediante Pearson

\begin{tabular}{|l|l|l|l|}
\hline \multicolumn{2}{|c|}{ Incubadoras Universitarias } & Incubadoras Universitarias & Modelo de Gestión \\
\cline { 2 - 4 } & Correlación de Pearson & 1 & $0.817^{* *}$ \\
\cline { 2 - 4 } & Sig. (bilateral) & & 0.002 \\
\cline { 2 - 4 } & Suma de cuadrados y productos vectoriales & 0.525 & 1.194 \\
\cline { 2 - 4 } & Covarianza & 0.053 & 0.119 \\
\cline { 2 - 4 } & $\mathrm{N}$ & 30 & 30 \\
\hline Modelo de Gestión & Correlación de Pearson & $0.817^{* *}$ & 1 \\
\cline { 2 - 4 } & Sig. (bilateral) & 0.002 & 12.487 \\
\cline { 2 - 4 } & Suma de cuadrados y productos vectoriales & 1.194 & 0.431 \\
\cline { 2 - 4 } & Covarianza & 0.119 & 30 \\
\cline { 2 - 3 } & $\mathrm{N}$ & 30 & 30 \\
\hline
\end{tabular}

Fuente. Elaboración propia: **. La correlación es significativa en el nivel 0,01 (2 colas). 
startups; los criterios de selección basada en la innovación tecnología influye positivamente al éxito de las startups; los mecanismos de financiamiento basado en el acceso a diversas fuentes influye positivamente al éxito de las startups; la vinculación universidad-empresa-gobierno con base en una plataforma de innovación facilita positivamente la comercialización exitosa de las tecnologías desarrolladas por las startups. En la figura 2, se muestra el marco conceptual de esta investigación y los cinco factores influyentes propuestas para la prueba empírica.

\section{Requerimientos de las incubadoras y star- tups en el Perú.}

La figura 3, muestra a las incubadoras de empresas que requieren diversos recursos tangibles e

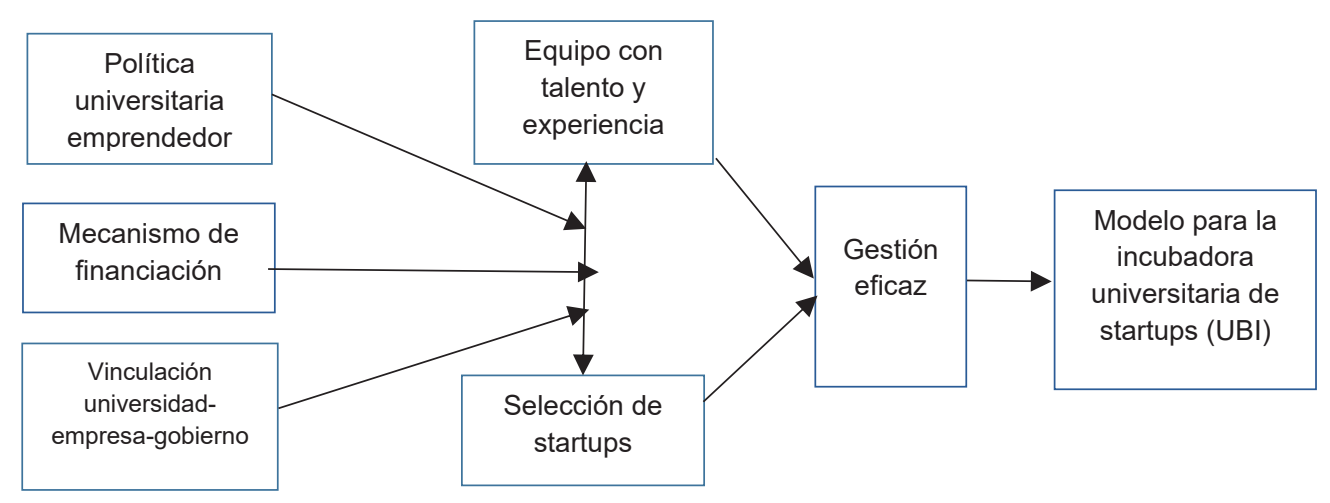

Figura 2. Marco conceptual de investigación del modelo.

Fuente: Elaboración propia.

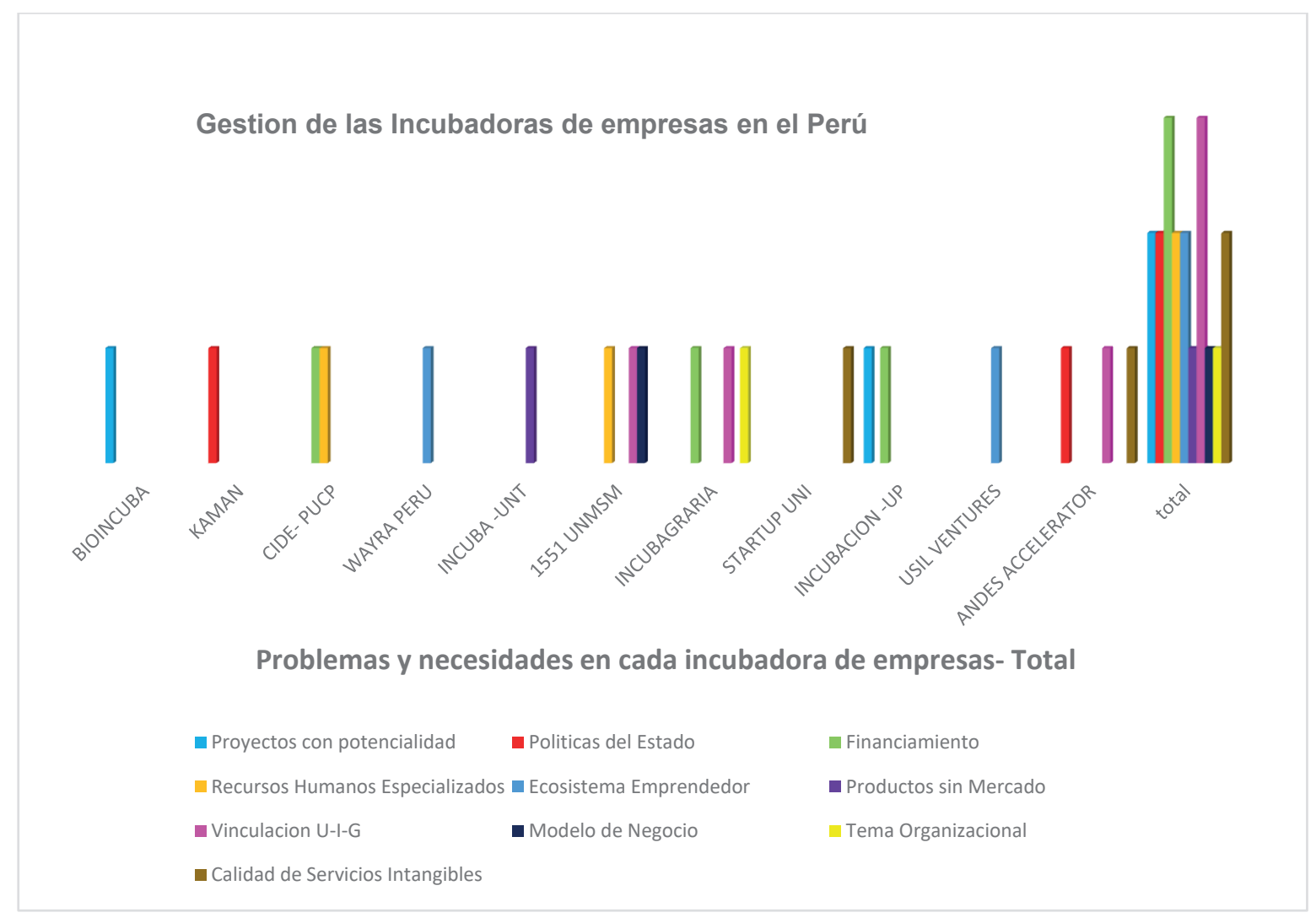

Figura 3. Problemas y necesidades en la incubación de empresas.

Fuente: Elaboración propia. 
intangibles para su funcionamiento, además los problemas de cada incubadora. El financiamiento es el factor más problemático, la vinculación universidad-empresa-gobierno es el factor de mayor necesidad.

La figura 4 muestra los requerimientos prioritarios de los startups, en la que predomina la asistencia en servicios intangibles; la capacitación, la asesoría y la mentoria son los más prioritarios.

Por los resultados se infiere que la gestión de las incubadoras debe considerar: la política que debe adoptar la universidad respecto al ecosistema de incubación; respecto a los recursos humanos, el tipo de personas que deben dirigir tanto las incubadoras y las startups; la selección de las startups para la incubación; el financiamiento de las incubadoras para apoyar la incubación de las startups y la incubación en colaboración para encontrar un mayor rendimiento de la producción tecnológica e innovación del ecosistema de incubación.

\section{MODELO DE GESTIÓN DE LA INCUBADORA UNIVERSITARIA DE STARTUPS}

Con la incubación universitaria de startups se puede transformar la producción intelectual universitario con ventaja competitiva, propiciando en profundidad la investigación y desarrollo, la innovación y el emprendimiento universitario para el desarrollo de los estudiantes, graduados, empleados, profesores, la universidad y el país. Las universidades en el Perú, deben patrocinar la incubadora universitaria de startups, similar a la incubadora de empresas universitarias (UBI). Mecanismo en que las universidades desarrollan y obtienen los resultados de la investigación, incluyendo las invenciones e innovaciones para la comercialización a través de la creación de startups (preferentemente nuevas empresas de base tecnológica). También propiciar la relación e interacción entre la academia, la industria, el gobierno, con el propósito de fomentar el proceso de comercialización, buscar incentivos económicos y fiscales del gobierno. Así mismo, propiciar el desarrollo económico, local, regional y nacional a través de la creación de empleos y la generación de utilidades. La propuesta del modelo de gestión de la incubadora universitaria de startups se muestra en la figura 5 .

La incubadora universitaria tiene como Visión "Ser el principal ecosistema tecnológico de alta calidad en la incubación de startups en el Perú"; y como Misión "Promover en la universidad ideas de negocios e incubar emprendimientos empresariales innovadores, apoyar la creación y desarrollo de startups de base tecnológica, brindándoles herramientas para el éxito

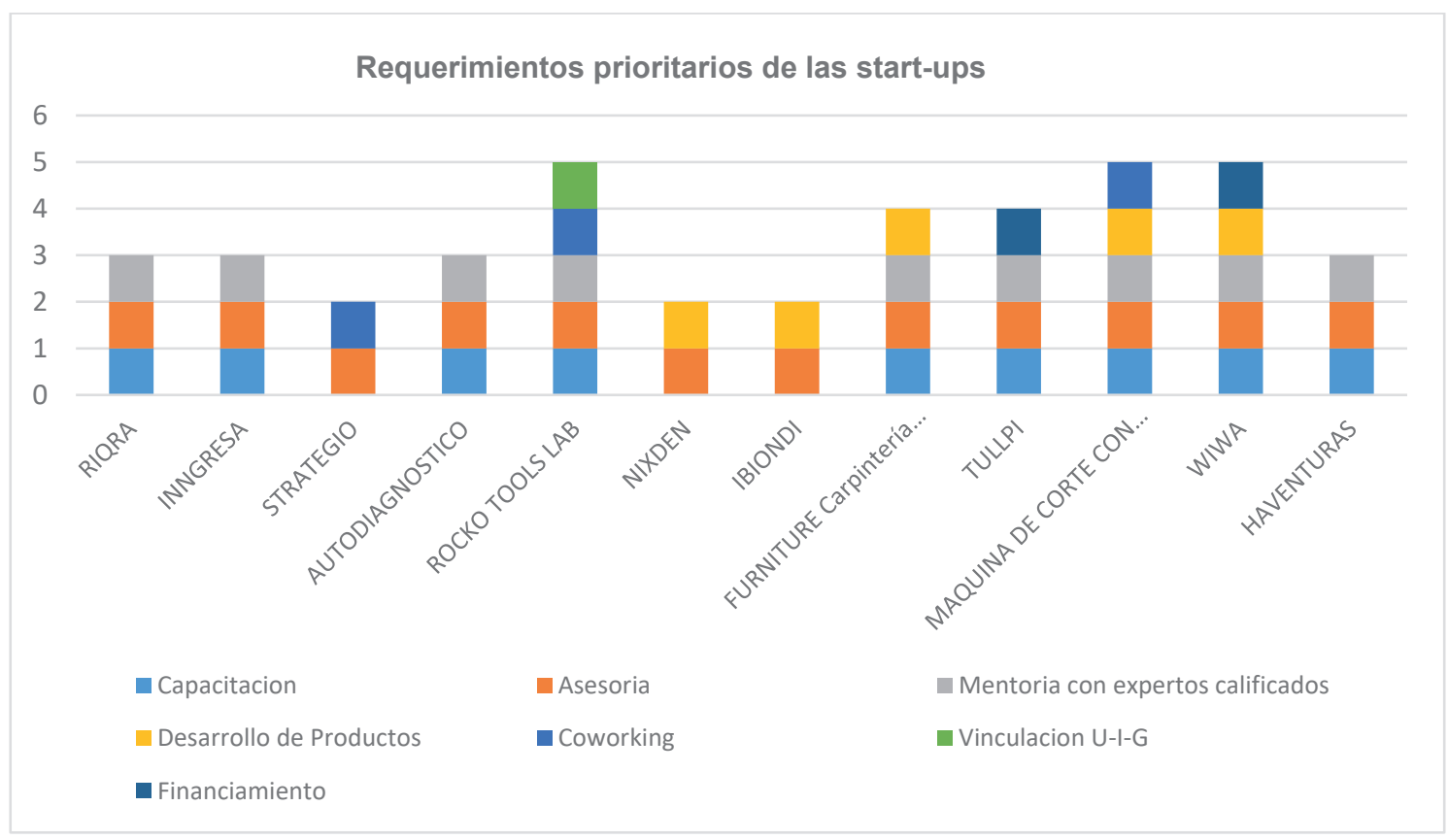

Figura 4. Requerimientos prioritarios de las startups.

Fuente: Elaboración propia. 
y su sostenibilidad". La estrategia de gestión se basa en desarrollar y decidir: en el entorno micro (la incubadora), el eficiente y eficaz trabajo sobre los factores críticos de éxito, como es contar con un equipo de gestión de primer nivel para la correcta selección de las startups; en el entorno meso (la universidad) propiciar una política universitaria viable para ser un ecosistema de innovación y emprendimiento, lograr vinculación entre la universidad-empresa y gobierno; buscar y lograr financiamiento para la incubadora y startups, con mecanismos más adecuados de entidades públicas y privadas. Las gestiones en los entornos micro y meso están en relación directa e indirecta con las políticas de entono macro (Estado), este último debe emitir la normatividad más conveniente para el propósito de innovación y emprendimiento de la universidad pública en el país. La incubadora, tiene como objetivos: crear, desarrollar y lograr el éxito de los startups de base tecnológica; crear y consolidar cultura de innovación y emprendimiento; contribuir con el desarrollo económico de la universidad, la región y el país.

Tiene como metas: el mejor rendimiento y éxito de la incubadora y startups, evaluadas mediante el impacto de ellas en la economía local, regional y nacional; la eficiencia, con el número de la creación y el crecimiento de nuevos startups; la sostenibilidad, con la tasa de supervivencia de startups graduadas.

Este modelo está constituido por la sumatoria positiva de 5 ejes principales: 1) Política universitaria emprendedor, para convertir la universidad actual en una universidad emprendedora, con marcadas actividades como: diseñar el ecosistema de innovación universitaria, donde el propósito, las actividades, la estructura, las personas y la cultura organizacional, tengan relación con los componentes de la incubadora, y contar con acciones estrategias emprendedoras bien definidas. 2) Dirección de la incubadora, mediante un equipo de personas asignadas o autoasignadas con habilidades y competencias específicas, quienes cumplen determinadas metas bajo la conducción de un líder y que tienen como política clara el trabajo en equipo, además que practican una serie de estrategias, procedimientos y metodologías para que logren las metas propuestas. 3) Selección correcta de startups, sustentada en tres criterios: que sea startups de base tecnológica, creada por estudiantes y docentes de la universidad, y sea admitida por la incubadora de empresas universitarias. 4)

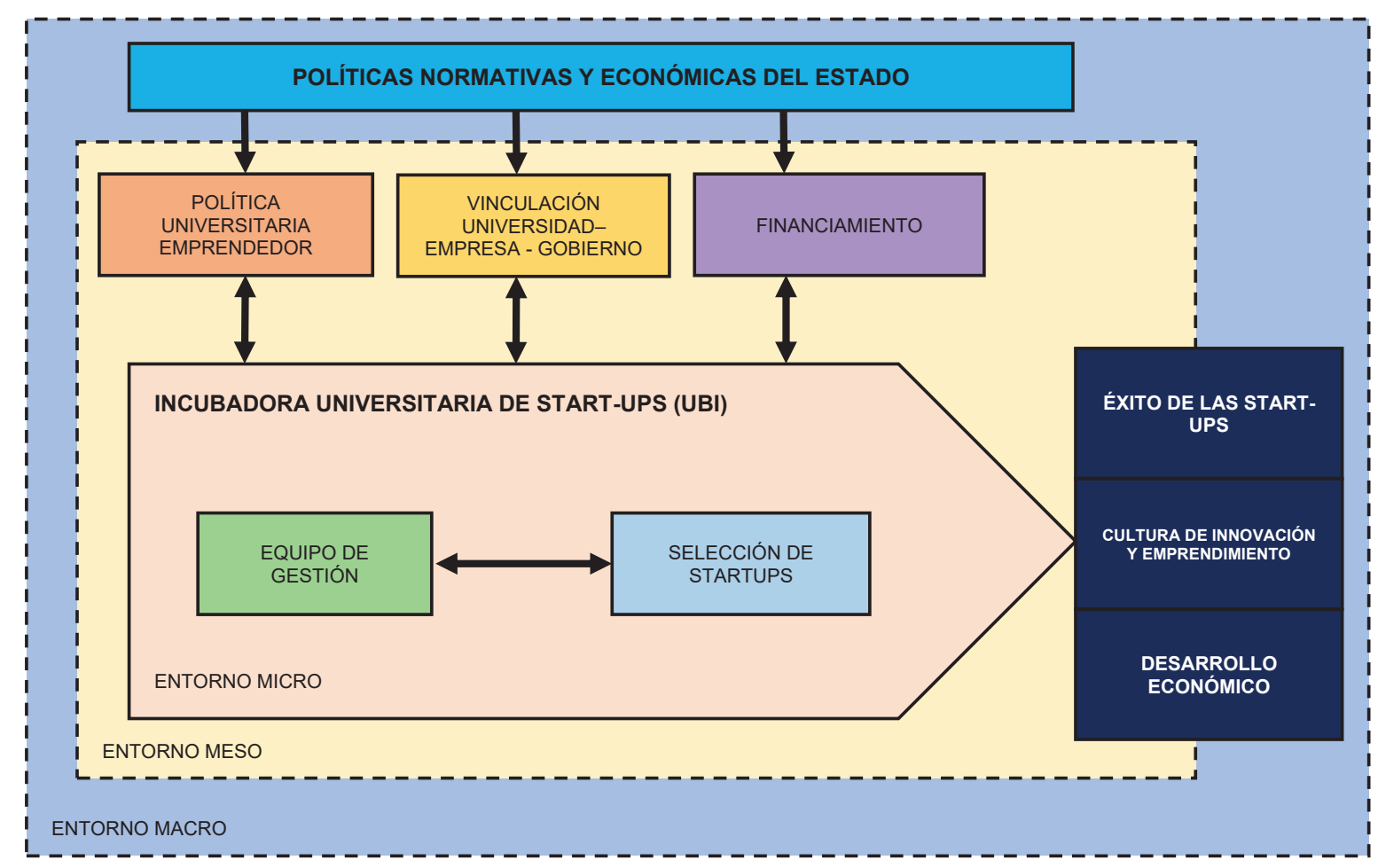

Figura 5. Modelo de gestión de incubadora universitaria de startups.

Fuente: Elaboración propia. 
Financiamiento, conforme a su característica y estructura de fuentes como: presupuesto público, fondos concursables, redes internacionales de incubadoras, recursos financieros privados, e ingresos propios. 5) Vinculación universidad-empresa-gobierno, mediante una plataforma interorganizacional, lograr fomentar innovación en países con economías en desarrollo como el caso del Perú, facilitar la comercialización de tecnologías desarrolladas por los startups incubadas exitosamente y permitir el incremento del número de publicaciones de artículos científicos y desarrollar las patentes y licencias.

\section{DISCUSIÓN Y CONCLUSIONES}

En este trabajo, se estudia y analiza la literatura sobre modelos de gestión en incubadoras de empresas, se desarrolla un diagnóstico del ecosistema de incubacion de empresas en el Perú, con el objetivo de conocer los factores que influyen el éxito de la incubacion de startups de base tecnológica; además, asimismo, se obtiene información y conocimiento sobre innovación, emprendimiento y desarrollo económico en las universidades.

Se identifica los principales problemas de incubacion de empresas siguientes: las diferencias por la forma de gestión, existen diferentes modelos (Grimaldi y Alessandro, 2005); el bajo rendimiento del equipo en las incubadoras; el flujo de conocimientos producido por los agentes endógenos y exógenos en la gestión de incubadoras de empresas tecnológicas no logran los resultados esperados (Rubin et al., 2015); en el proceso de incubacion interactivo en colaboración interorganizacional, no se conocen los impactos de los factores contextuales regionales (McAdam et al., 2016); en los aceleradores, las características del conocimiento empírico, teórico, y los conducentes es escasa (Pauwels et al., 2016); falta analizar el papel de las incubadoras de empresas universitarias, en sus estrategias de incubacion e innovación para apoyar el desarrollo de las empresas (Wonglimpiyarat, 2016). Y los hallazgos de las encuestas en el ecosistema de incubacion en el Perú, evidencia la falta de un modelo de gestión eficaz en las incubadoras de empresas universitarias, se puede concluir que los resultados encontrados, son consistentes en este estudio.

El modelo de gestión de la incubadora universitaria de startups en el Perú propuesta, fue evaluado y validado por 30 personalidades expertas en el ámbito del Perú; por las incubadoras: 1 directora, 4 gerentes generales, 3 coordinadores, 1 director ejecutivo, 3 jefes de emprendimiento, y 1 especialista en innovación; por los startups: 6 CEO, 3 CMO, 4 CTO, 3 GG y 1 cofundador, de quienes se puede considerar que sus opiniones son de valor; esta evaluación de los expertos se validó estadísticamente mediante el Modelo de Regresión y Correlación de Pearson, donde la correlación de Pearson Rp $=0.817$, y un Valor $p$ $=0.002$, este resultado permite inferir que como el Valor $p=0.002<0.05$, se acepta la propuesta. Este resultado es un primer aporte a la literatura y que las futuras investigaciones pueden tener como referencia; se afirma que el ecosistema universitario de incubacion de startups en el Perú todavía es insipiente, este aspecto es una limitación en las encuestas que se ha realizado, además permite opinar la falta de estudios sobre modelos de gestión de incubadoras universitarias con métricas que midan el éxito de los startups.

\section{REFERENCIAS}

[1] Ankrah, S.; AL-Tabbaa, O. (2015). Universitiesindustry collaboration: A systematic review. Scandinavian Journal of Management, 31, 387-408.

[2] Arena, M.; Bengo, I.; Calderini, M.; Chiodo, Ch. (2018). Unlocking finance for social tech start-ups: Is there a new opportunity space? Technological Forecasting \& Social Change, 127, 154-165.

[3] Baraldi, E.; Ingemansson, M. (2016). Identifying new dimensions of business incubation: A multilevel analysis of Karolinska Institute's incubation system. Technovation, 50-51, 53-68.

[4] Bergek, A.; Norrman, Ch. (2008). Incubator best practice: A framework. Technovation, 28, 20-28.

[5] Bruneel, J.; Ratinho, T.; Clarysse, B.; Groen, A. (2012). The Evolution of Business Incubators: Comparing demand and supply of business incubation services across different incubator generations. Technovation, 32, 110-121.

[6] Correia da Silva, L.; Vasconcellos, A.; Campos da Silva, F.; Oliveira, J. (2016). Strategic Management Method for the Incubation Process of Industrial Companies: Case Study of the Tooling Industry in Brazil. Procedia CIRP $41,129-134$.

[7] Daskalopoulou, I.; Liargovas, D.; Petrou, A. (2010). A comparative study of business incubators and technoparks in the EU. International Journal of Innovation and Regional Development, 2, 198-220. 
[8] Good, M.; Knockaert, M.; Soppe, M.; Wright, M. (2019). The technology transfer ecosystem in academia. An organizational design perspective. Technovation, 82-83, 35-50.

[9] Grimaldi, R.; Alessandro, G. (2005). Business incubators and new venture creation: an assessment of incubating models. Technovation, 25, 111-121.

[10] Kalar, B.;Antoncic, B. (2015). The entrepreneurial university, academic activities and technology and knowledge transfer in four European countries. Technovation, 36-37, 1-11.

[11] Liu, Y.; Huang, Q. (2018). University capability as a micro-foundation for the Triple Helix model: The case of China. Technovation, 7677, 40-50.

[12] Matt, M.; Feng, M. (2010). Management of university incubators in China and in France: a comparative analysis. Int. J. Entrepreneurship and Innovation Management, 11, 282-300.

[13] McAdam, M.; Miller, K.; McAdam, R. (2016). Situated regional university incubation: A multilevel stakeholder perspective. Technovation, 50-51, 69-78.

[14] Mrkajic, B. (2017). Business incubation models and institutionally void environments. Technovation, 68, 44-55.

[15] OECD (2013). An international benchmarking analysis of public programmes for high-growth firms. OECD LEED programme, Paris.

[16] Pauwels, Ch.; Clarysse, B.; Wright, N. VanHove, J. (2016). understanding a new generation incubation model: The accelerator. Technovation, 50-51, 13-24.

[17] Rajalo, S.; Vadi, M. (2017). University-industry innovation collaboration: Reconceptualization. Technovation, 62-63, 42-54.
[18] Rubin, T.; Helge, T.; Stead, A. (2015). Knowledge flowin Technological Business Incubators: Evidence from Australia and Israel. Technovation, 41-42, 11-24.

[19] Soltanifar, E.; Keramati, A. (2012). An innovative model of business management in knowledgebased organisations: the case of the business incubators. Int. J. Business Innovation and Research, 6, 573-596.

[20] Somsuk, N.; Laosirihongthong, T. (2014). A fuzzy AHP to prioritize enabling factors for strategic management of university business incubators: Resource-based view. Technological Forecasting \& Social Change, 85, 198-210.

[21] Stal, E.; Andreassi, T. Fujino, A. (2016). The role of university incubators in stimulating academic entrepreneurship. RAI Revista de Administração e Inovação, 13, 89-98.

[22] Studdard, N. (2006). The effectiveness of entrepreneurial firm's knowledge acquisition from a business incubator. International Entrepreneurship and Management Journal, 2, 221-225.

[23] Wonglimpiyarat, J. (2016). The innovation incubator, university business incubator and technology transfer strategy: The case of Thailand. Technology in Society, 46, 18-27.

[24] Yong, W.; Kyun, Y.; Rami, Ch. (2018). Experience-based human capital or fixed paradigm problem? CEO tenure, contextual influences, and corporate social (ir) responsibility. Journal of Business Research, 90, 325-333. 